P. Alonso Lecue ${ }^{1,4}$, J. Rodriguez Carrio ${ }^{5}$, D. Prieto-Peña ${ }^{1,3}$, V. Portilla ${ }^{1,3}$, R. Blanco ${ }^{1,3}$, A. Corrales ${ }^{1,3}$, J. M. Cifrián-Martínez ${ }^{1,4,6}$, R. López-Mejías ${ }^{1}, M$. A. González-Gay ${ }^{1,3,6,7},{ }^{1}$ IDIVAL, Research Group on Genetic Epidemiology and Atherosclerosis in Systemic Diseases and in Metabolic Bone Diseases of the Musculoskeletal System, Santander, Spain; ${ }^{2}$ Hospital Universitario Marqués de Valdecilla, 'López Albo' Post-Residency Programme, Santander, Spain; ${ }^{3}$ Hospital Universitario Marqués de Valdecilla, Department of Rheumatology, Santander, Spain; ${ }^{4}$ Hospital Universitario Marqués de Valdecilla, Department of Pneumology, Santander, Spain; ${ }^{5}$ Faculty of Medicine, Universidad de Oviedo, Deparment of Functional Biology, Immunology Area, Oviedo, Spain; ${ }^{6}$ Universidad de Cantabria, School of Medicine, Santander, Spain; ${ }^{7}$ Faculty of Health Sciences, University of the Witwatersrand, Cardiovascular Pathophysiology and Genomics Research Unit, School of Physiology, Johannesburg, South Africa

Background: Interstitial lung disease (ILD) is one of the most significant complications of connective tissue diseases (CTD), leading to an increase of the morbidity and mortality in patients with CTD [1]. A specific T cell subset termed angiogenic $\mathrm{T}$ cells (TAng), that promote endothelial repair and revascularization, have been involved in the pathogenesis of CTD [2-4]. However, to the best of our knowledge, no information regarding the role of TAng in CTD-ILD ${ }^{+}$is available.

Objectives: To study, for the first time, the potential role of TAng related to vascular damage in CTD-ILD ${ }^{+}$

Methods: Peripheral venous blood was collected from 40 patients with CTD-ILD ${ }^{+}$ and three comparative groups: 44 CTD-ILD patients, 21 idiopathic pulmonary fibrosis (IPF) patients and 20 healthy controls $(\mathrm{HC})$. All subjects were recruited from the Rheumatology and Pneumology departments of Hospital Universitario Marqués de Valdecilla, Santander, Spain. Quantification of TAng was performed by flow cytometry. TAng were considered as triple-positive for CD3, CD31 and CXCR4.

Results: Patients with CTD-ILD+ exhibited a significantly lower TAng frequency than CTD-ILD patients $(p<0.001)$. Similar results were obtained when patients with CTD-ILD ${ }^{+}$were compared with $\mathrm{HC}(\mathrm{p}=0.004)$ although no difference was observed between CTD-ILD ${ }^{+}$and IPF. In addition, a significant increase of TAng frequency was shown in patients with CTD-ILD in relation to IPF patients $(p<0.001)$, while no difference was observed between CTD-ILD' and HC.

Conclusion: Our results reveal a decrease of TAng frequency related to vascular damage in CTD-ILD ${ }^{+}$. Furthermore, we disclose that the presence of ILD is associated with lower TAng frequency.

REFERENCES:

[1] Expert Rev Clin Immunol 2018;14(1):69-82.

[2] Circulation 2007:116(15):1671-82.

[3] Ann Rheum Dis 2015 74(5):921-7.

[4] PLoS One 2017;12(8):e0183102.

Acknowledgements: Personal funds, VP-C: PREVAL18/01 (IDIVAL); SR-M: RD16/0012/0009 (ISCIII-ERDF); LL-G: INNVAL20/06 (IDIVAL); RP-F: START PROJECT (FOREUM); RL-M: Miguel Servet type I CP16/00033 (ISCIII-ESF).

Disclosure of Interests: Verónica Pulito-Cueto: None declared, Sara Remuzgo Martinez: None declared, Fernanda Genre: None declared, Belén Atienza-Mateo: None declared, Victor Manuel Mora-Cuesta: None declared, David Iturbe-Fernández: None declared, Leticia Lera-Gómez: None declared, Raquel Pérez-Fernández: None declared, Pilar Alonso Lecue: None declared, Javier Rodriguez Carrio: None declared, Diana Prieto-Peña: None declared, Virginia Portilla: None declared, Ricardo Blanco Speakers bureau: Abbvie, Pfizer, Roche, Bristol-Myers, Janssen and MSD, Consultant of: Abbvie, Pfizer, Roche, Bristol-Myers, Janssen and MSD, Grant/research support from: Abbvie, MSD and Roche, Alfonso Corrales: None declared, Jose Manuel Cifrián-Martínez: None declared, Raquel López-Mejías: None declared, Miguel A González-Gay Speakers bureau: Pfizer, Abbvie, MSD, Grant/research support from: Pfizer, Abbvie, MSD

DOI: 10.1136/annrheumdis-2021-eular.2676

\begin{tabular}{|l|l|}
\hline AB0027 & INCREASED CIRCULATING CD39+FOXP3+CD4+TREG \\
CELLS IN EARLY RHEUMATOID ARTHRITIS FACILITATE \\
THE ANTIINFLAMMATORY ACTION OF METHOTREXATE
\end{tabular}

L. Nuño ${ }^{1}$, A. Villalva ${ }^{1}$, M. Novella-Navarro ${ }^{1}$, I. Monjo ${ }^{1}$, D. Peiteado ${ }^{1}$, S. GarcíaCarazo $^{1}$, A. Puig-Kröger ${ }^{2}$, A. Balsa ${ }^{1}$, M. E. Miranda-Carus ${ }^{1} .{ }^{1}$ Hospital Universitario La Paz, Rheumatology, Madrid, Spain; ${ }^{2}$ Gregorio Marañón Hospital, Immunology, Madrid, Spain

Background: Methotrexate (MTX) remains the first line of treatment in Rheumatoid Arthritis $(R A)^{1,2}$. Inhibition of AICAR transformylase by MTX results in augmented release of adenine nucleotides to the extracellular space ${ }^{1}$; these are rapidly hydrolysed by the combined action of ectonucleotidases CD39 and CD79 rendering the antiinflammatory agent adenosine ${ }^{1}$. CD39, the rate-limiting enzyme in this cascade, is highly expressed by a subset of human FoxP3+CD4+ regulatory T cells (Treg39+) ${ }^{2-}$ ${ }^{4}$ and MTX may act synergistically with Tregs in the control of inflammation.

Objectives: To study the expression of CD39 on circulating Treg cells of untreated early Rheumatoid Arthritis (ERA) patients and its relation with the ex vivo effect of MTX. Methods: Peripheral blood was drawn from 22 DMARD- and steroid- naïve ERA patients with a disease duration $<24$ weeks, 15 longstanding RA patients (LRA, disease duration $>2$ years) and 37 age and gender-matched healthy controls (HC). LRA patients were receiving low-dose weekly MTX and were naïve for biologicals. 10 ERA patients who had achieved remission 12 months after initiating MTX donated blood for a second time (ERA-R). The frequency of Treg and Treg cell subsets was assessed by flow cytometry. CD4+CD25+CD127- (total T reg) CD4+CD27+CD127-CD39+ Treg (Treg39+) and CD4+CD25-CD39- responder T (Tresp39-) cells were isolated by Ficoll-Hypaque, followed by sorting. The suppressor potency of Tregs was assessed in cocultures of isolated Tregs with Tresp, established at different Treg/Tresp ratios. Proliferation was determined by CFSE dilution; cytokine secretion was measured by ELISA of culture supernatants.

Results: As previously described ${ }^{5}$, ERA but not LRA patients demonstrated a superior frequency of circulating Treg (CD4+CD25+CD127-FoxP3+) cells. In addition, the proportion of Tregs that expressed CD39 (Treg39+) was significantly increased in ERA but not LRA. Total ERA Tregs were significantly more potent suppressors of proliferation, TNF $\alpha$ and IFN $\gamma$ secretion when compared with HC or LRA Tregs, and this difference was partially and significantly abrogated in the presence of adenosine deaminase, or the adenosine A2A receptor (A2AR) antagonists DMPX (3,7-dimethyl-1-propargylxanthine) or ZM 241385, but not in the presence of the adenosine A1 receptor antagonist DPCPX (8-cyclopentyl-dipropylxanthine). When MTX was added to the culture medium, the suppressor potency of total Tregs was further enhanced in all 3 groups of patients, and this enhancement was significantly higher in ERA total Treg/Tresp39- cocultures as compared with HC or LRA total Treg/ Tresp39- cocultures. The effect of MTX was also partially and significantly abrogated by adenosine deaminase, DMPX or ZM 241385 but not by DPCPX. We then tested the suppressor potency of isolated Treg39+ together with the enhancer effect of MTX on this potency, and observed that there were no longer differences among ERA, LRA and $\mathrm{HC}$; this further suggests that the differences observed in assays using tota Tregs can be attributable to the increased Treg39+ proportions present in ERA. The frequency and function of ERA-R Treg cells were not different from HC or LRA Tregs. Conclusion: The suppressor action of CD39+Tregs is mediated at least in part by adenosine trough A2AR ligation, and the superior suppressive potency of total ERA Tregs is associated with their higher proportion of TregCD39+ cells as compared with HC or LRA. In addition, the augmented suppressor effect observed in the presence of MTX is partly mediated by an increased adenosine production acting on A2AR and is more marked in ERA patients reflecting again their higher proportion of Treg39+ cells. This indicates that MTX cooperates with Treg39+ cells in the control of inflammation.

REFERENCES:

[1] Montesinos MC, et al. Arthritis Rheum. 2007.

[2] Peres RS, et al. Proc Natl Acad Sci U S A. 2015.

[3] Deaglio S, et al. J Exp Med. 2007.

[4] Borsellino G, et al. Blood. 2007.

[5] Benito-Miguel M, et al. J Immunol. 2009.

Acknowledgements: FIS PI 20/00141; FIS RD16/0012/0012; Fondo Europeo de Desarrollo Regional (FEDER), unrestricted research grant from Gebro Pharma Disclosure of Interests: Laura Nuño: None declared, Alejandro Villalva: None declared, Marta Novella-Navarro: None declared, Irene Monjo: None declared, Diana Peiteado: None declared, Sara García-Carazo: None declared, Amaya Puig-Kröger: None declared, Alejandro Balsa Grant/research support from: BMS Gebro Pharma, Maria-Eugenia Miranda-Carus Grant/research support from BMS, Gebro Pharma

DOI: 10.1136/annrheumdis-2021-eular.2680

\section{AB0028 EXPRESSION OF FATTY ACID TRANSPORTERS AND FREE FATTY ACID UPTAKE IN CD8+ T CELLS IN AUTOIMMUNE ARTHRITIS PATIENTS}

S. Keck ${ }^{1}$, F. V. Kraus ${ }^{1}$, H. M. Lorenz ${ }^{1}$, M. Souto-Carneiro ${ }^{1} .{ }^{1}$ University Hospital Heidelberg, Department of Rheumatology, Heidelberg, Germany

Background: RA CD8+ T cells (CD8s) have been shown to vastly contribute to tissue damage and RA disease activity by presenting and maintaining an active effector phenotype promoting autoinflammation. In order to meet their high metabolic requirements RA CD8s alter not only their glycolytic and glutaminolytic profiles, but also rely on increased lipid metabolism characterized by increased free fatty acid (FFA) uptake and lipid biosynthesis.

Objectives: In order to classify the importance of lipid metabolism in RA CD8s in terms of initiation and perpetuation of effector functions and therefore exploring potential novel therapy targeting points or biomarkers additional investigations have to been made. By analysing FFA uptake and the expression of free fatty acid transporters (FAT) upon TCR-stimulation in RA, PsA, SpA patients and healthy controls $(\mathrm{HC})$, we aimed to further characterize the importance of RA CD8s' lipid metabolism. Methods: Blood samples from 23 RA patients, 22 SpA patients, 20 PsA patients and $7 \mathrm{HC}$ recruited at Heidelberg University Hospital were processed via negative magnetic separation selection to obtain purified CD8s. CD8s were cultured for 72 hours in $[1,6-13 C]$ glucose containing medium in the presence of anti-CD3 and anti-CD28. FFA uptake was measured by Palmitate-Bodipy 488 staining (ThermoFisher), the expression of FAT CD36, FABP4 and GPR84 besides CD69, CD36, CD45RA, CD3 and CD8 were assessed by FACS analysis. 
Ethic approval NR: S-096/2016.

Results: The intake of the FFA palmitate by CD8s was higher in all patients groups than in $\mathrm{HC}(\mathrm{p}<0,05)$. In all three diseases' stimulated CD8s, unlike in the HC group, the expression of GPR84 was inversely correlating to the expression of CD69 (Spearman $r<-0,62)$. In patient's stimulated CD8s the expression of GPR84 tended to be lower in RA and PSA effector memory (EM) CD8s. In RA and $\mathrm{HC}$ the expression of FABP4 tended to be lower in the naive CD8+ subset when compared to the effector subset. HC naive and effector CD8+ subsets had a higher expression of CD36 than in the patient groups. In RA and SPA patients the expression of FAT correlated with clinical variables. In RA DAS28 and CRP inversely correlated with CD36 (MFI), and disease duration with FABP4 as well. In SPA CRP and BASDAl inversely correlated with FABP4 while disease duration had a negative correlation with CD36 (MFI). GPR84 (MFI) had an inverted relationship to BASDAI. While the BMI was directly correlated with the expression of CD36 in RA, this relationship was inverted in SPA. Conclusion: A high free fatty acid uptake seems to characterize autoimmune arthritis CD8+ T cells. The gain of effector functions appears to be connected to changes in the expression of different fatty acid transporters on the surface of CD8+ T cells. The correlation between the expression of fatty acid transporters and clinical parameters (specially disease activity scores) in RA and SPA suggests that they could potentially be used as biomarkers for disease activity and progression. Acknowledgements: We thank all the individuals involved in the study for their participation.

Disclosure of Interests: None declared

DOI: 10.1136/annrheumdis-2021-eular.3069

\section{Innate immunity in rheumatic diseases}

\section{AB0029 THE METABOLIC HIERARCHY OF IMMUNE PROCESSES IN HUMAN MONOCYTES}

P. L. Krauß ${ }^{1}$, F. Buttgereit ${ }^{1}$, T. Gaber ${ }^{1}$, M. Pfeiffenberger ${ }^{1}$, Y. Chen ${ }^{1}$, T. Buttgereit ${ }^{2}$. ${ }^{1}$ Charite Universitaetsmedizin Berlin, Rheumatology and Clinical Immunology, Berlin, Germany; ${ }^{2}$ Charite Universitaetsmedizin Berlin, Dermatology, Venerology, and Allergology, Berlin, Germany

Background: At sites of inflammation, monocytes carry out specific immunological functions while facing challenging bioenergetic restrictions.

Objectives: Here, we investigated the potential of human monocytes to adapt under conditions of reduced energy supply by gradually inhibiting oxidative phosphorylation (OXPHOS) under glucose free conditions.

Methods: We modelled this reduced energy supply with myxothiazol, an inhibitor of mitochondrial respiration, at 0,2 and $4 \mathrm{pmol} / 10^{6}$ cells to decrease mitochondrial ATP production for $0 \%, 25 \%$ and $66 \%$ under glucose free conditions. For the three energy levels, we assessed (i) phagocytosis of FITC-labelled E.coli using flow cytometry, (i) production of reactive oxygen species (ROS) through NADPH oxidase (NOX) as determined by VAS2870-sensitive OCR using a Clark-type electrode, (iii) ATP generation and steady state level using a Clark-type electrode and luminometric assessment (iv) expression of surface activation markers CD16, CD80, CD11b, HLA-DR and (v) production of the inflammatory cytokines $\mathrm{IL}-1 \beta, \mathrm{IL}-6$ and TNF- $\alpha$ using flow cytometry in peripheral blood-derived human monocytes with and without LPS-stimulation.

Results: As a prerequisite for our study, we demonstrate that human monocytes survived strong inhibition of mitochondrial respiration without any sign of apoptosis as determined by flow cytometry. As a result of the inhibition of OXPHOS, we demonstrate a reduction of VAS2870-sensitive OCR (ROS production through NOX), ATPase-dependent OCR and ATP steady-state levels. Focusing on immune function, we observed that phagocytosis and the production of IL-6 were the least sensitive to reduced energy supply while surface expression of CD11b, HLA-DR, production of TNF- $\alpha$ and IL-1 $\beta$ were most affected by inhibition of OXPHOS.

Conclusion: Our data demonstrate an energy-dependent hierarchy of immune functions in monocytes, which may represent a potential therapeutic target in monocyte-mediated inflammatory diseases.

Disclosure of Interests: None declared

DOI: 10.1136/annrheumdis-2021-eular.794

\section{AB0030 $\quad$ BETA BOSWELLIC ACID BLOCKS INNATE IMMUNE RESPONSES IN MULTIPLE OA JOINT CELLS}

E. Franco-Trepat ${ }^{1}$, A. Lois-Iglesias ${ }^{1}$, A. Alonso-Pérez ${ }^{1}$, M. Guillán-Fresco ${ }^{1}$, M. López-Fagúndez ${ }^{1}$, J. P. Cerón-Carrasco ${ }^{2}$, A. Pazos-Pérez ${ }^{1}$, A. Crespo-Golmar ${ }^{1}$, A. Jorge-Mora ${ }^{1}$, S. Belén Bravo ${ }^{3}$, R. Gómez ${ }^{1} .{ }^{1}$ Institute IDIS, Musculoskeletal Pathology Group, Santiago de Compostela, Spain; ${ }^{2}$ Universidad Católica de Murcia (UCAM), Bioinformatics and High Performance Computing (BIO-HPC), Murcia, Spain; ${ }^{3}$ Institute IDIS, Proteomics Unit, Santiago de Compostela, Spain

Background: Osteoarthritis (OA) incidence has skyrocketed in the last decade and yet a definitive treatment has still to be found. This worldwide disease is depriving our society from their life quality and has become a grave economic burden.
Research on anti-inflammatory tools has been done on traditional Asian medicine. Boswellic acid is a plant-derived molecule from the Boswellia species that has shown to prevent cartilage loss in an OA mouse model[1]. However, the specific mechanism of action is still unclear. The activation of innate immune receptors, such Toll-like receptor 4 (TLR4) has been involved in chondrocyte-mediated inflammatory responses and OA development. Although, boswellic acid has shown an inhibitory effect on TLR4-mediated inflammatory responses little is known about its role on TLR4-mediated chondrocyte inflammatory and catabolic responses.

Objectives: Determine the ability of beta boswellic acid (BBA) to block TLR4-mediated innate immune responses in chondrocytes and synoviocytes.

Methods: In silico

The binding affinity of beta boswellic acid (BBA) to TLR4 complex signalling was determined by optimized docking algorithm in the BIO-HPC Research Group facilities.

In vitro

Cellular proteome and secretome profiling (LC-MALDI/TOFF) was used to study inflammatory pathways induced by the agonist of TLR4 (LPS [100ng/ml]) and IL1R (IL1 $\beta[0.1 \mathrm{ng} / \mathrm{ml}])$. The effect of BBA on TLR4-mediated innate immune responses was determined by RT-PCR, Western Blot and ELISA in primary human OA chondrocytes (hOC), murine ATDC5 chondrocytes, human synoviocytes (SW982) and primary human osteoblasts (hOB). Cell viability was tested using the methyl-thiazolyl-tetrazolium (MTT) reagent. Nitric oxide production in cell culture media was assessed by Griess reaction. Green Malachite Assay was used to semi-quantify the whole phosphoproteome.

Ethics

This study was approved by the CEIC (CAEIG 2014/310).

Results: Cellular proteome and secretome profiling validated the activation of TLR4 and IL1R signalling by LPS and IL1 $\beta$ ligands and revealed an enrichment in innate immune responses (NF-K $\beta$, NLRP3, MMPs, Interleukins, etc).

Non-toxic doses of BBA [0.5-1000nM] prevented the activation of TLR4 in multiple articular joint cells and inhibited TLR4 \& IL1R-dependant innate immune responses at the mRNA and protein level such as inflammatory factors IL6 NOS2, COX2, LCN2, MMP1, -3, -9, -13 and ADAMTS4, among others. Furthermore, NF-K $\beta / / K B \alpha$ and NLRP3/PYCARD/IL1 $\beta$ axis were also severely inhibited after BBA treatment. Moreover, these results were validated by in silico docking analysis that showed BBA interacted with TLR4/NF-K $\beta$.

Conclusion: We prove that BBA inhibit TLR4 \& IL1R -dependent innate immune responses in multiple human joint cells (Figure 1). We show that NF-K $\beta$ \& NLRP3 signalling, both associated to OA, are blocked (mRNA and protein) after BBA treatment (Figure 1).

Our data support previous studies showing the prevention of cartilage loss in an OA animal models by BBA might come from its ability to inhibit TLR4 signalling In the clinical practice of rheumatologists, Boswellia Serrata could be a useful nutraceutical to manage OA inflammation due to its content in BBA.

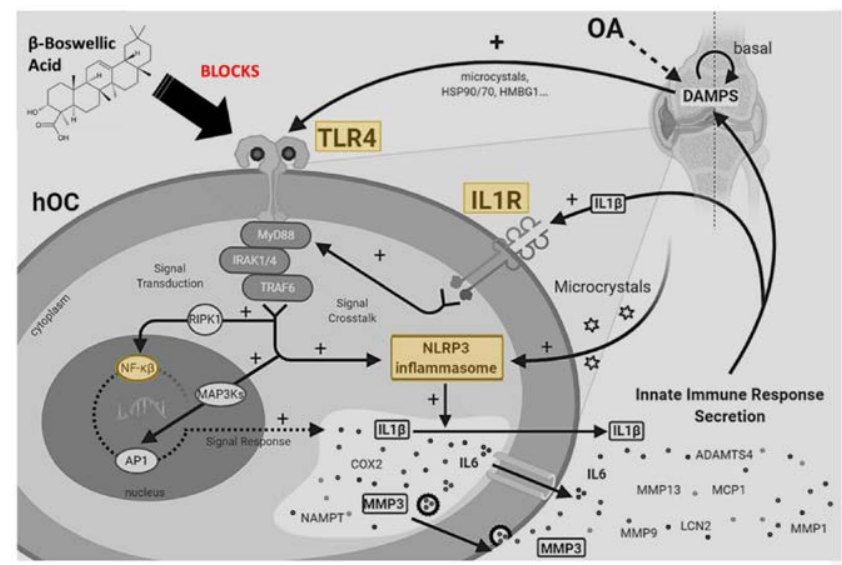

Figure 1.

\section{REFERENCES:}

[1] Wang, Q.; Pan, X.; Wong, H.H.; Wagner, C.A.; Lahey, L.J.; Robinson, W.H.; Sokolove, J. Oral and topical boswellic acid attenuates mouse osteoarthritis. Osteoarthr. Cartil. 2014, 22, 128-132, doi:10.1016/j.joca.2013.10.012.

Acknowledgements: Eloi Franco-Trepat and Ana Lois-Iglesias contributed equally to this work.

This research has been funded by the non-profit FER (Fundación Española de Reumatologia /Spanish Foundation of Rheumatology) through the project "Búsqueda de nuevos fármacos bloqueantes de la inflamación asociada a TLR4 en condrocitos humanos artrósicos".

Disclosure of Interests: None declared

DOI: 10.1136/annrheumdis-2021-eular.1889 